\title{
Interactive comment on "Monitoring of solar spectral ultraviolet irradiance in Aosta, Italy" by Ilias Fountoulakis et al.
}

\section{Anonymous Referee \#1}

Received and published: 12 August 2020

\section{General remarks:}

The manuscript by Fountoulakis et al. describes a new dataset of UV radiation, measured by a Bentham spectroradiometer in northern Italy. The paper includes descriptions of the instrument's characterization, corrections applied to the measurements, the traceability of calibrations, the data's uncertainty, comparisons with the QASUME reference spectroradiometer, and available data products. The good agreement between measurements of QASUME and the author's instrument gives confidence in the good quality of data from the latter. While data of this instrument have been published before, the manuscript focuses on a new data version ("Level 2") and its uncertainty budget. The work is therefore appropriate for the journal of Earth System Science Data. Considering that spectral UV data with a well documented uncertainty are sparse, the new 
dataset is of value for the scientific community. I downloaded one of the datasets from the website indicated in the paper (https://doi.org/10.5281/zenodo.3934324) and compared a few spectra with my own model calculations. I found the data easy to use and did not encounter a problem.

Methods and materials are generally described in sufficient detail in the manuscript, however, there are many minor issues (see Specific Remarks below) that need to be addressed before the manuscript can be published. In general, when referring to an "expanded uncertainty", please also provide the coverage factor. It is likely $\mathrm{k}=2$, corresponding to a confidence level of $95 \%$, but this is often not clear.

The paper is well structured but in need for extensive copy-editing. While the level of English is sufficient to understand the text, there are numerous grammatical errors (e.g., the placement of adverbs), which should be corrected before publication. In my review, I only point out language issues that could be missed by the copy editor. I trust that the remaining errors will be fixed during the production stage. The formatting of Table 1 and 2 is unusual. The format should also be corrected during production.

Specific remarks:

L22: "expanded uncertainty": provide the coverage factor (likely $k=2$ )

Figure 1: It would be much better to show a topographical map instead of a political map.

L174: "As already discussed the Bentham5541 is traceable to world reference QASUME." I would say the measurements are traceable to the scale of spectral irradiance established by PTB. At least the chain shown in Figure 5 starts with PTB. QASUME is only used for QA/QC, but data are not scaled to match QASUME's measurements. The role of QASUME should be better described.

Section 3.1: either use the term "dark current" or "dark signal" but not a mixture of both. L195: Why would the dark current depend on "the level of the intensity of the incoming 
light"? The dark current is presumably measured with a physical shutter closed, or perhaps determined by scanning below $280 \mathrm{~nm}$ where no solar radiation can be detected at the Earth's surface (presuming that there is no stray light). So the "level of the intensity of the incoming light" should be irrelevant.

L203: Why is the uncertainty related to the recorded signal 10 negligible? When approaching the detection limit, 10 is affected by noise, so the uncertainty goes to infinity as the detection limit is approached.

L206: Eq. (1) does no look correct. It should be $I 1=(I 0$ - D) / A. The dark current should be measured at the same amplification as the "light" current. So if there is no light entering the PMT, 10 and D should be identical. Since the dark current is not zero (e.g., because of Schott noise) and scales with the amplification, I1 can only be zero when no radiation is falling on the diffuser if $I 0$ and $D$ are divided by the same factor $A$. If the authors use indeed their Eq. (1) to calculate I1, the error would be small if $I 0$ is much higher than D. However, there would be a significant error at very low light levels when 10 is only somewhat larger than D.

Line 224: $y$ is presumably the correction factor $\mathrm{cf}$, and $\mathrm{x}$ is presumably temperature theta in ${ }^{\circ} \mathrm{C}$. If so, the equitation should be written: of $=a{ }^{*}$ theta $+b$. Also, I find it awkward to use a symbol with two letters to describe a factor. Why not use "c" instead of "cf"?

Line 226: If theta is the symbol for temperature, it should also be used in Eq. (2).

L230: This is only justified if the PTFE diffuser material used by Y\&S is the same as that used by the authors. Is this the case? What is the evidence that both diffuser materials have the same temperature dependence?

L231: "Since the 125th Day of the Year (DOY), in May of 2017". Just say: "Since 5 May 2017 ..."

L255 and L258: I am surprised that distances as close as 5 or $7 \mathrm{~cm}$ between lamp and 
diffuser are considered. At $5 \mathrm{~cm}$, a $1 \mathrm{~mm}$ uncertainty in the lamp-diffuser distance results in an uncertainty of about a $4 \%$ in the irradiance at the diffuser. Since the authors like to detect differences of less than $0.5 \%$, the lamp-diffuser distance would have to be reproducible by $0.12 \mathrm{~mm}$. Is the coupling between the lamp housing and the diffuser really that reproducible? The author should comment on the trade-off between short distance (= low noise) and long distance (= less uncertainty from distance inaccuracies and less uncertainty from heating the diffuser).

Figure 3 and lines 280, 281, 287 Figure 3 does not show a "correction factor". Instead it shows the cosine error of the diffuser, expressed in measured angular response divided by the ideal (=cosine) response, for illumination by a point source and for isotropic illumination. The term "correction factor" is highly confusing because it implies that the values shown in Figure 3 are the values by which solar measurements have to be scaled to correct for the diffuser's cosine error. However, such a correction would also depend on wavelength, as correctly discussed in Section 3.3.2.

L282: "distributed uniformly in the horizon" > "isotropic"

Figure 4c: I don't understand why the error increases sharply between $85^{\circ}$ and $88^{\circ}$ considering that solar radiation becomes more diffuser over this angular range and the lack of a large spike in the angular response at these angles, according to Figure 3. It seems to me that the spike shown in Figure $4 \mathrm{c}$ is a result of interpolation artifacts of the cosine error due to the fact that the cosine error at $90^{\circ}$ is infinite.

Section 3.5. The experiments described in this section do not characterize nonlinearity. Non-linearity means that the output of an instrument (in this case the signal I2) varies linearly with the input (i.e., spectral irradiance). An experimental setup for testing non-linearity could, for example, involve a light source whose intensity can be changed over a wide range (ideally the same range as covered by solar radiation) plus a beam splitter that would direct half of the light towards the Bentham and the other half on a second radiometer whose linearity has been confirmed by other means. By comparing

Interactive

comment 
the signal of the Bentham with that of the secondary (linear) radiometer, departures from linearity can be determined. (There are also other means to characterize a radiometer for non-linearity, but the test above illustrates the principle sufficiently.) PMTs in photon-counting mode tend to be non-linear if two photos arrive within the dead-time of the counter, but this is not the case here.

Instead, the experiments described in Section 3.5 tested something different. They determined whether the responsivity of the system changes (either permanently or temporarily) if the system's PMT is temporarily exposed to either high light levels, or is producing large currents due to a unreasonably high high-voltage setting. The term "hysteresis" comes to mind describing the effect but it not the ideal term either. Perhaps using descriptive headings would be the best. For example, "Section 3.5, Non-linearity" could be replaced with "Section 3.5. Change in responsivity after exposure to high radiation levels". Likewise, Subsection 3.5.1 and 3.5.2 could be replaced with "Change in responsivity during usual operating conditions" and "Change in responsivity after exposure to unusually high radiation levels", respectively. The word "non-linearity" in the text of Section 3.5. should also be replaced with a more descriptive term such as "change in responsivity".

L367: The maximum photocurrent of a PMT of the type used by the authors is typically $1000 \mathrm{nA}$. It should be noted that the PMT was operated well beyond the recommended range.

L368, sentence starting with "Two possible ...": What does this sentence refer to? The period when photocurrent was $>15000$, or the period after adjusting the HV to $400 \mathrm{~V}$ resulting in photocurrents $<500 \mathrm{nA}$ ? Also the phrase "of similar instrument" is strange. It suggests that problems resulting from PMT overexposure are about to be described in general terms. Instead, the rest of paragraph addresses only problems observed with the Bentham.

Line 370: I don't understand "the day following the intensity of the recorded signal". 
L395: I don't understand "However, in this case the response of the AAO gradually increased while we were measuring the lamps irradiance at noon." AAO standards for "Aosta-Saint Christophe". Do you mean the responsivity of the system located at AAO increased? Also "gradually" implies that you measured the lamp several times, and there was a clear trend in responsivity. Was this the case?

L407: The maximum photocurrent during these experiments was apparently $2500 \mathrm{nA}$, resulting in a $3 \%$ effect. However, in line 368 it is noted that photocurrents as high $20000 \mathrm{nA}$ were measured before July 2006. This suggests that measurements during this period are likely affected by saturation. Is this the reason why the dataset (e.g. Fig. 9) does not include measurements prior to July 2006?

\section{L440: Explain acronym "KS"}

Section 4.1: Explain that spectral measurements are traceable to the scale of spectral irradiance PTB. I assume that the PTB has realized their scale more than once. The specific PTB scale used for the measurements of the network should be referenced.

L466: "in units of electrical intensity (nA)" > "in units of nA"

L482: Why $320 \mathrm{~nm}$ ? 315-400 $\mathrm{nm}$ is now the standard range for UV-A.

Line 490 references Mayer and Kylling, 2005 for the UVSPEC model while line 304 references Emde et al., 2016. Were two different model versions used? If not, perhaps both papers could be cited on line 304 and the reference in line 490 could be omitted.

L523: "corresponds to the minimum response of the diffuser" The diffuser does not have a responsivity. What's likely meant is that the responsivity of the system is smaller when the diffuser's temperature is lower. Please clarify.

L535: "even the Level 1.5 spectra are of good quality." The sentence suggests that Level 1.5 data are less accurate than Level 1.0 data, which is likely not the case. Rephrase or delete "even" at the least. 
L554: "by the company" What company? ; "quality certificate." > "calibration certificate."

L558 - L593: The text in these paragraphs is very hard to follow. There are changes ESSDD in the calibration, which were later reversed when new information became available. I guess this information is of little relevance for the average reader and only of importance to the authors for documenting the adjustments in calibration factors. Hence, there may be no need to improve the text. Still, I am wondering whether the information could be presented in an easier-to-digest format. Perhaps a table could be added that describes the reasons for the differences in Level 2 and Level 1 data for the different periods discussed in the text.

Caption Figure 8: The sentence "Shaded area represents $\pm 2 \%$ from perfect agreement between Bentham5541 and QASUME" is likely not correct. The $\pm 2 \%$ range is indicate by two lines. The shaded areal is likely "the combined expanded uncertainty of the Bentham5541 Level 2 and the QASUME spectra for each intercomparison" as indicated in line 613.

L630: Add "for most years" after "There is a clear improvement of the results when the Level 2 dataset is used." The difference in the results of both instruments is worse for Level 2 data in 2006, 2011, and 2013.

Section 5.1.5.: Non-linearity was not tested. The sentence should be changed to: "The responsivity of the Bentham5541 does not change by more than $0.5 \%$ if the system is exposed for short times to high radiation levels that may occur during clear skies in summer months. The resulting uncertainty is set to zero because it is part of the uncertainty from instrument instability (Sect. 5.1.3)."

L750: I recall from reading Section 3.5 that the responsivity my change by up to $0.5 \%$ after overexposure. So the uncertainty cannot not be $0 \%$. If the uncertainty is part of another uncertainty component, this should be stated.

Caption Table 2: According to the caption, numbers are "standard uncertainties". I sus- 
pect that this is incorrect and that expanded uncertainties for $\mathrm{k}=2$ are shown. But I may be wrong. In either case, since Table 2 presents the final uncertainty budget, expanded uncertainties should be shown. All number should also be larger than the expanded uncertainties shown in Table 1 because of additional uncertainty components affecting solar measurements.

L773 - L778: Also uncertainties in this section should be "expanded" uncertainties.

L790: "is linear, even for very high signal levels." No. There was no real test of nonlinearity. Only changes in responsivity following overexposure were tested - see my comments above. Having said this, I believe that the system is indeed linear. Otherwise, the comparison between the Bentham and QASUME would have indicated differences as a function of solar irradiance. This could be mentioned.

L796 - 800: It is not clear whether the uncertainties mentioned here are expanded uncertainties or not.

L861 and L52: Change year from 2013 to 1994.

Specific comments to data available at https://doi.org/10.5281/zenodo.3934324:

The paper includes the link https://doi.org/10.5281/zenodo.3934324 that points to the actual data. The associated website on ZENODO is well organized, however, a few shortcomings and errors should be fixed:

- Data files are compressed in zip files. The naming convention of these zip files should be added to the website.

- The readme.txt file that describes the data files includes the sentence: "* Spectral scans begin at $290 \mathrm{~nm}$ and usually end at $400 \mathrm{~nm}$. However, scans may also end at $400 \mathrm{~nm}$." I presume "may also end at $400 \mathrm{~nm}$." should be "may also end at $500 \mathrm{~nm}$."

- The same readme file also includes the line: "Columns 6 - 446: Irradiance (in Watt $/ \mathrm{m}^{\wedge} 2 / \mathrm{nm}$ ) at wavelengths $290-500 \mathrm{~nm}$ respectively with a step of $0.25 \mathrm{~nm}$ " The

(1) 
number of wavelengths between 290 and 500 in $0.25 \mathrm{~nm}$ steps should be 841 , not 446 .

Language:

ESSDD

Language - General:

(Important!) Change "response" (i.e. the term describing the ratio of signal and irradiance) to "responsivity" throughout the paper. See for example:

Interactive https://en.wikipedia.org/wiki/Responsivity

"dependence from" > "dependence on"

"In a different day" > "On a different day"

"of the order of" > "in the order of"

"data was" > "data were" (data is plural)

"which mediates" > "which lies" or just delete, or reword sentence.

"Huelsen" > "Hülsen"

Always include a space between number and unit (e.g., $570 \mathrm{~m}>570 \mathrm{~m}$ )

"statistic noise" > "statistical noise"

Language - Specific:

L10: "in the North-western" > "in the North-Western region"

L14: "moreover" > "also"

L18: "The used Quality" > "The Quality"

L21: "consist one of" > "consist of one of"

L37: "exposed to more or to less" > "exposed to either more or to less"

L40: Delete "and on time" 
L46: "the North and the South hemisphere in spring in the 1980s," > "both hemispheres during spring since the 1980s"

L48: "also experienced" > "were also observed"

L69: Delete "However,"

L83: "certifies" > "confirms" (To certify something, you typically have to be accredited by a licensing board or a standards organization.)

L96: tall > high

L128: "consists from" > "consists of"

L149: "driven" > "coupled" or "guided"

L305: Angstrom > Ångström

Section 3.4: The word "head" for describing the metal piece at the end of the fiber is awkward. I suggest "termination". Line 333: Change "on the first head and exits the fiber through a second aperture on the second head." to "at one end and exits the fiber through a second aperture on the other end."

L342: The sentence "in order to detect possible azimuthal dependence of the response, which would show that there is a problem with the azimuth response," is a tautology. Just delete the second part of the phrase.

L345: "bellow" > "below"

L349, 353: "bubble" > "bubble level"

L354, 689: "After begin" > "After" L377: "at early morning," > "in the early morning"

L442: "basis at the facilities of the PMOD- WRC" > "basis to PMOD-WRC"

L448: "the three working standard 200 Watt lamps" > "the three 200 Watt working standards" 
L467: Rephrase "divided with the used level of amplification."

L479, 504: "DOY 125 of 2017" > "5 May 2017"

L539: "characterized" > "classified"

L549: "however exceed" > "exceed"

L667: "standard throughout the years" > "identical for all years"

Interactive

comment

L680: "to the accredited" > "at accredited"

L697: "Sect. 3.5 resulted that" > "Sect. 3.5 suggests that"

L725: delete "and certify"

L733: "Thus, a strict calculation of the uncertainty would demand to take" > "Thus, a rigorous calculation of the uncertainty would require to take"

Caption Table 1: "(in \%) o the Level 2" > "(in \%) for Level 2"

L782: "range 290 - $500 \mathrm{~nm}$ " > "range of $290-500 \mathrm{~nm}$ "

L789: "proved through" > "confirmed by"

L790: "It has been also proved that" > "It has also been shown that"

L795: "have significant contribution to" > "contribute significantly to"

L812: "when at the UV index for at least 15 days of the month is available." > "when the noon-time UV index is available for at least 15 days per month."

Interactive comment on Earth Syst. Sci. Data Discuss., https://doi.org/10.5194/essd-2020-116, 2020. 